\title{
Drainless Parotidectomy Using Tissue Fibrin Sealant - A Case-Control Study
}

\author{
Oded Cohen ${ }^{1}$, Hen Chaushu ${ }^{1}$, Keren Hod ${ }^{1}$, Tzur Kirshenbaum ${ }^{1}$, and Avi Khafif ${ }^{1}$ \\ ${ }^{1}$ Assuta Medical Center
}

April 14, 2021

\begin{abstract}
Objectives: Drainless parotidectomy has been reported infrequently, mostly in patients undergoing limited partial parotidectomies. Evicel is a fibrin sealant (FS) glue whose use has not been reported in head and neck surgeries so far. The aim of the study was to investigate the impact of drainless parotidectomy using FS on the surgical outcome. Design: A retrospective matched case-control series. Settings: A single academic center, Participants: All cases of patients who underwent drainless parotidectomies, including deep lobe tumors and revision surgeries, were compared to age, sex, body mass index and tumormatched controls in which a suction drain was inserted. Main outcome measures: length of hospital stay (LOS). Secondary outcomes included post-operative seroma and related complications. Results: A total of 123 patients (41 cases and 82 controls) were included in the study. Pre-operative and intra-operative characteristic did not differ significantly between the FS group and controls. A borderline significance was found for surgery type as the FS group had higher rates of total parotidectomy compared with the control group $(25.0 \%$ vs. $10.5 \%, \mathrm{p}=0.054)$. LOS was significantly shorter in the FS group (1.0 \pm 0.3 vs. $1.5 \pm 0.6$ days, $\mathrm{p}<0.001)$. The rates of post-operative seroma, aspirations, local infection and post-operative antibiotic treatment were all lower in the FS group compared with the control group, but did not reach statistical significance. Conclusion: A drainless parotidectomy using the EVICEL FS is safe, reduces LOS, and may reduce post-operative seroma and its associated complications. This procedure may be applied to deep parotid tumors, parapharyngeal involvement and revision surgery.
\end{abstract}

\section{Abstract:}

Objectives: Drainless parotidectomy has been reported infrequently, mostly in patients undergoing limited partial parotidectomies. Evicel is a fibrin sealant (FS) glue whose use has not been reported in head and neck surgeries so far. The aim of the study was to investigate the impact of drainless parotidectomy using FS on the surgical outcome.

Design: A retrospective matched case-control series

Settings: A single academic center

Participants: All cases of patients who underwent drainless parotidectomies, including deep lobe tumors and revision surgeries, were compared to age, sex, body mass index and tumor-matched controls in which a suction drain was inserted.

Main outcome measures: length of hospital stay (LOS). Secondary outcomes included post-operative seroma and related complications.

Results: A total of 123 patients (41 cases and 82 controls) were included in the study. Pre-operative and intra-operative characteristic did not differ significantly between the FS group and controls. A borderline significance was found for surgery type as the FS group had higher rates of total parotidectomy compared with the control group ( $25.0 \%$ vs. $10.5 \%, \mathrm{p}=0.054)$. LOS was significantly shorter in the FS group (1.0 \pm 0.3 vs. $1.5 \pm 0.6$ days, $\mathrm{p}<0.001)$. The rates of post-operative seroma, aspirations, local infection and post-operative 
antibiotic treatment were all lower in the FS group compared with the control group, but did not reach statistical significance.

Conclusion: A drainless parotidectomy using the EVICEL FS is safe, reduces LOS, and may reduce postoperative seroma and its associated complications. This procedure may be applied to deep parotid tumors, parapharyngeal involvement and revision surgery.

\section{Key points:}

- Fibrin sealant is biological glue which have been used in different head and neck surgeries

- Drainless parotidectomy has been published in selected cases, yet has been not adopted widely

- The current series includes both deep lobe tumors and revision cases in a consecutive series of patients

- While decreasing length of stay and post-operative rates, no increase in complications was noted

- Drainless parotidectomy using FS seems feasible even for complicated parotid surgeries and should be considered by experienced head and neck surgeons

\section{Introduction}

Use of fibrin sealants (FS) has been shown to reduce post-operative secretion and length of hospital stay (LOS) in different types of head and neck surgeries, mostly thyroidectomies, but also parotidectomies and neck dissections $\left[{ }^{1},{ }^{2},{ }^{3},{ }^{4},{ }^{5},{ }^{6},{ }^{7}\right]$.

While FS use in thyroid surgery is well established, with several randomized controlled trials and meta analyses suggesting improvement in the post-operative course $\left[{ }^{1},{ }^{8}\right]$, data on the outcome of FS-based drainless parotid surgeries remains limited $\left[3{ }^{3},{ }^{4},{ }^{5},{ }^{9},{ }^{10}\right]$. Previous reports on FS-based drainless parotidectomies have shown improved outcomes compared with the traditional approach of drain insertion, resulting in shorter LOS and reduced post-operative seroma rates $\left[{ }^{4},{ }^{5}\right]$. Despite the encouraging results in published data, the vast majority of parotid surgeons would still insert a drain in virtually all of their patients. Possible explanations for this are concerns regarding secretion of saliva from the remaining parotid tissue as well as the potential dead space. Therefore, additional studies on the outcome of FS use in parotid surgery are still needed in order to consider drainless parotidectomy with FS as the standard of care.

FS contain fibrinogen and thrombin frozen solutions. These components act to form a clot which adheres to the wound surface and seals small blood vessel.

The aim of this matched case-control study was to evaluate the impact of drainless parotidectomy using FS on LOS, post-operative seroma and related complications in a consecutive group of patients who underwent parotidectomies in our department.

\section{Patients and Data}

After an approval by our institution's review board, a retrospective study of all patients above 18 years of age who underwent parotidectomy (including total, superficial or partial parotidectomy) at our department between 2016 and 2020 was performed.

Prior to November 2019 all patients were drained following parotid surgery. From November 2019 onwards a shift in practice has been made and all patients were treated with Evicel (Evicel Airless Spray Accessory, Omrix Biopharmaceuticals Ltd, Ethicon Inc, Somerville, NJ) FS without drainage regardless of the extent of parotid surgery. No other changes were made in the procedure, instrumentation or surgical technique. The extent of surgery was determined at the surgeon's discretion, based on the tumor's location, cytology, size, etc.

The FS group included all patients in whom an Evicel FS was placed in the surgical bed at the end of the operation and a drain was not inserted. The control group included patients in whom a drain (BIOVAC closed wound drainage system, Biometrix, Jerusalem, Israel) was inserted at the end of the operation. The control group patients were matched (eliminating significant difference) for age, sex, body mass index (BMI), surgery type, tumor type and size (see below) with the FS group patients. Exclusion criteria included patients 
with missing pertinent data, patients who underwent neck dissection in addition to parotid surgery, patients with a history of neck irradiation and patients in whom both drainage and FS were used at the end of the surgery.

\section{Surgical procedure}

All surgeries were performed by the same high-volume head and neck surgeon (A.K.). An individualized modified mini-Blair incision ${ }^{[11}$ ] was used in all patients. The relevant facial nerve branches or trunk were identified according to the location of the tumor and the surgical needs. The tumor was excised with clean borders and non-involved parotid tissue was preserved. The FS group received 2 cc of Evicel, prepared according to the manufacture's specifications. After meticulous hemostasis, Evicel was sprayed on the surgical bed using a designated jet syringe system (Figure 1). When a substantial mass of parotid gland was removed and a significant post-operative cosmetic deficit was expected, the sternocleidomastoid muscle was detached from its insertion to the mastoid and sutured to the parotid gland prior to the placement of the Evicel glue, in order to decrease potential dead space and cosmetic deficit at the surgical bed.

\section{Study outcomes}

The main outcomes of the study were LOS, post-operative seroma/salivary collection, and the incidence of local infection. A diagnosis of post-operative seroma was based on an impression of fluctuation in the surgical bed by the surgeon, confirmed by needle aspiration of fluids through the suture line. Our team's approach for treatment of post-operative seroma was as follows: minimal seromas were followed without intervention. Initial intervention was needle aspirations. If a seroma still recurred following repeated aspirations, then a vacuum drain was inserted $\left[{ }^{12}\right]$.

\section{Data collection}

All medical records were reviewed and the following data were extracted: Pre-operative data included demographics (age and sex), diabetes and smoking status (as reported at admission), BMI, and prior parotid surgery (revision). Intra-operative data included parotidectomy type (partial, superficial or total), performance of a neck dissection (for exclusion from the analysis), tumor location (superficial, deep lobe, tail or accessory parotid gland), length of surgery (minutes) and insertion of surgical drains vs. FS. Hospitalization data included LOS (days), days with drain, total amount of drained fluids, post-operative complications, post-operative antibiotics and visual analog scale (VAS) on post-operative day 1 and at discharge. Data on the final pathology (tumor type) and final tumor size $(\mathrm{cm})$ were also collected. Post-hospitalization data included post-operative seroma or infection as documented in outpatient visits, seroma aspiration (number and total amount) if performed, prescription of antibiotics, insertion of a drain and readmission.

\section{Statistical analysis}

Categorical variables are reported as numbers (percentages), and continuous variables are reported as mean \pm standard deviation (SD). Continuous variables were tested for normal distribution using histograms and Q-Q plots. Categorical variables were compared using chi-squared test or Fisher's exact test and continuous variables - by Mann-Whitney test or t-test, as appropriate. Revision surgeries were excluded from surgery type and tumor size analysis. A two-tailed $\mathrm{p}<0.05$ was considered statistically significant. Analyses were performed with SPSS version 22 (IBM Corporation, Armonk, NY).

\section{Results}

A total of 123 patients (41 cases and 82 controls) were included in the study. Patient demographics and pre-operative characteristics are presented in Table 1 . The mean age of the study cohort was $50.8 \pm 12.9$ years, with a female predominance $(69 / 123,56.1 \%)$. No statistically significant differences between the FS and control group were found for any of the pre-operative characteristic.

Comparison of intraoperative characteristics and the final pathology is presented in Table 2. No significant differences were found between the groups regarding tumor location and final pathology. A borderline significance was found for surgery type as the FS group had higher rates of total parotidectomies compared with 
the control group $(25.0 \%$ vs. $10.5 \%, \mathrm{p}=0.054)$. Revision surgery was performed in 5 patients $(12.2 \%)$ in the FS group and $6(7.3 \%)$ in the control group. Parapharyngeal involvement was found in 2 patients $(4.9 \%)$ in the FS group and $6(7.3 \%)$ in the control. The most common pathology was pleomorphic adenoma in both groups $(65.9 \%$ and $50.0 \%$ of patients in the FS and control group, respectively). Malignant tumors were found in $4.9 \%$ and $3.7 \%$ of patients in the FS and control group, respectively.

The mean LOS in the FS group was significantly lower than that in the control group (1.0 \pm 0.3 vs. $1.5 \pm 0.6$ days; $\mathrm{p}<0.001$, Figure 2). Within the control group, the mean number of days with a drain was $1.6 \pm 0.6$, with a mean volume of $19.5 \pm 15.9 \mathrm{cc}$ of drainage per hospitalization day. Two patients were discharged with their drain, which was removed after 2 days in our outpatient clinic. No differences in mean VAS score was noted between the FS and the control groups - either on post-operative day $1(0.9 \pm 1.1$ vs. $1.0 \pm 0.9, \mathrm{p}=0.645)$ or on the day of discharge ( $0.8 \pm 1.0$ vs. $0.6 \pm 0.8, \mathrm{p}=0.509)$.

Complications were minimal and included transient facial nerve palsies as well as salivary and serous collections and infections. No permanent complications occurred in any of our patients regardless of the use of FS or drain. Comparisons of post-operative complications are presented in Table 3. No significant difference was found. The rate of post-operative seroma aspirations and delivery of post-operative antibiotics were lower in the FS group compared with the control group but did not reach statistical significance (Table 3). In the FS group 3 patients were each aspirated twice, and in the control group 12 patients were aspirated 1-3 times $(\mathrm{p}=0.282)$. The mean volume of aspirations was similar in both groups $(1.0 \pm 3.6 \mathrm{cc}$ in the FS group and $1.0 \pm 3.2 \mathrm{cc}$ in the control group, $\mathrm{p}=0.299)$.

\section{Discussion}

The aim of the current study was to evaluate the feasibility of drainless parotid surgery following the application of FS to the surgical bed. Our results suggest that drainless parotidectomy following application of FS is safe, reduces LOS and may reduce seromas. The inclusion of deep lobe tumors, parapharyngeal tumors and revision surgeries with no significant change in outcome suggests that the same manner of drainless surgery may also be applied in these patients. In agreement with previous studies supporting the role of FS following parotid surgery, we suggest that it should be considered as an alternative to the conservative use of drains following parotid surgery.

The use of FS has increased in recent years, including in the head and neck region $\left[{ }^{1},{ }^{2}, 3,{ }^{3},{ }^{5},{ }^{6},{ }^{7}\right]$. Yet, articles describing this procedure should be divided into those that examine the impact of FS use on drain output $\left[{ }^{2},{ }^{13},{ }^{14}\right]$, and those that refer to drainless surgery using FS $\left[{ }^{4},{ }^{6},{ }^{5},{ }^{9}\right]$. Regarding the former, a randomized controlled trial by Maharaj et al. has shown that the use of FS significantly reduced wound drainage by $30 \%$ and post-operative seroma from $22.7 \%$ to $3.6 \%\left[{ }^{2}\right]$. Previous studies have found that FS may reduce wound complications such as salivary fistula and hematoma $\left[{ }^{14}\right]$. The aforementioned publications have encouraged the principal surgeon (A.K) to start using FS in head and neck surgeries, including parotidectomies.

The first to question the necessity of drains in parotid surgery was Patel in $2006\left[{ }^{13}\right]$, yet this was employed on a selective group of patients. Concerns of leaving the parotid bed undrained are probably based on the rich blood supply and secretive nature of the gland. Interestingly, use of FS was feasible and safe in drainless mastectomy, which also involves a secretive gland rich in fat tissue $\left[{ }^{15}\right]$. Our study's result support the report by Congilio et al. $\left[{ }^{16}\right]$ on successful drainless outpatient parotidectomy in a selected group of patients. Conboy et al. described their experience in 20 patients who underwent drainless parotidectomy using FS $\left[^{6}\right]$. Two patients required aspirations due to post-operative seroma, and no other complications were noted. Chorney et al. investigated the impact of FS on wound complications in 100 patients $\left[{ }^{3}\right]$. While no suction drain was used in the FS group, a passive drain was used in $8.7 \%$ of patients in the FS group. Although there were no differences in wound complications between the groups, both groups had exceedingly higher rates of infection - $23.9 \%$ in the FS group and $16.7 \%$ in the drain group - compared to the rates of infection reported by other studies, including the current one. Chudek et al. reported that the use of FS has allowed for a drainless surgery, and resulted in an absolute risk reduction for seroma/salivary leak, as well as shortening the LOS $\left.{ }^{5}\right]$. However, as all patients underwent partial parotidectomies, while revision surgeries and deep lobe tumors 
were excluded, no conclusion could be drawn on FS use in these patients. The FS group in our study had a higher prevalence of deep lobe tumors compared to the control group (borderline significance), yet no difference was found in post-operative complications between the two groups. Furthermore, our cohort also included tumors involving parapharyngeal space as well as revision surgeries. Therefore, the current study further strengthens the findings by Chudek et al., suggesting that FS should be considered as an alternative for traditional suction drains even in complex parotid surgeries.

In recent years, the investigation of surgical outcome has shifted from safety-focused reports (i.e. complications, etc.) to safety and quality of life $\left[{ }^{17}\right]$. In the head and neck field this shift has been mostly prominent in thyroid and parathyroid surgery, encouraging reports on drainless surgery and minimally invasive approaches or on remote access approaches eliminating the cervical scar $\left[{ }^{1},{ }^{18},{ }^{19}\right]$. This rational was supported by recent studies that have emphasized the role of such scars on the quality of life of patients $\left[{ }^{20}\right]$. In parotid surgery this progress has been mild at best. Parotidectomy via a modified Blair incision and insertion of a suction drain is still considered the gold standard for treatment. The greatest change in the evolution of parotid surgery was the introduction of a modified/partial superficial parotidectomy, which leaves as much normal parotid tissue as possible and removes the tumor with a cuff of normal parotid tissue around it $\left[{ }^{17},{ }^{21}\right]$. Other modifications for incisions, including minimal skin incisions $\left[{ }^{11}\right]$ or cosmetic incisions $\left[{ }^{22}\right]$ have seldom been suggested. Studies using self-assessment scores have shown that surgical drains are associated with a significant increase in patients' anxiety and pain as well as reduced comfort $\left[{ }^{23}\right]$. Similar to the aforementioned studies on the impact of surgical neck scars $\left[{ }^{20}\right]$, studies on the impact of drains on patients' quality of life and post-operative recovery should be undertaken, which may further encourage a change in the surgical paradigm, as experienced by our group and others $\left[{ }^{4}, 5\right]$.

Finally, the decrease in post-operative LOS is also an important consideration when implementing drainless surgery. Most importantly, it reduces the exposure of patients and their families to nosocomial infections and improves patient satisfaction. While the mean LOS in our control group was 1.5 days, a US nationwide study $(\mathrm{n}=66,914)$ has reported a mean LOS of 2.5 days $\left[{ }^{24}\right]$. Another recent US nationwide study has found that a LOS [?]2 days was an independent risk factor for readmission within 30 days following parotidectomy $\left[{ }^{25}\right]$. Considering that the cost of a hospitalization day is USD [?]9000 $\left[{ }^{24},{ }^{25}\right]$ compared with the cost of FS $(\sim 300 \$)$, this cost difference should also be taken as a major factor for implementing drainless FS parotid surgery.

Our study has several limitations. As a retrospective study it is based on the quality of documentation of postoperative complications and patient adherence to follow-up. This has also prevented us from prospectively evaluating the impact of drain on patient post-operative satisfaction and quality of life. Although ours is one of the largest studies to report drainless parotidectomies, it is possible that a larger cohort would have had an impact on the statistical significance of the observed trends in post-operative outcomes. As our series included all consecutive patients operated on before and after the implementation of FS, selection bias based on case selection was not relevant, yet inherent selection bias of retrospective design remains. A minor limitation relates to generalizability of the findings given that it is a single surgeon experience. Drain device usage maybe country-specific, as well as hospital admission policy, observation practices and costing which may differ by geographies.

\section{Conclusions}

Drainless parotidectomy using the FS is safe, enables reducing LOS, and may reduce post-operative seroma and its associated complications. This procedure may also be applied to patients with deep parotid tumors, parapharyngeal involvement and revision surgery, further encouraging its use as an alternative for traditional drain insertion.

\section{References}

1. Samraj K GK. Wound drains following thyroid surgery. Cochrane Database Syst Rev . 2007;17(4). doi:10.1002/14651858.CD006099.pub2.www.cochranelibrary.com 
2. Maharaj M, Diamond C, Williams D, Seikaly H HJ. Tisseel to reduce postparotidectomy wound drainage: randomized, prospective, controlled trial. J Otolaryngol . 2006;35(1):36-39.

3. Chorney SR, Ryan JT. Fibrin sealant and parotidectomy wound complications in 100 patients. Eur Arch Oto-Rhino-Laryngology . 2019;276(9):2621-2624. doi:10.1007/s00405-019-05540-3

4. Cunniffe HA, Wong BLK, Hilger AW BO. Drain - free parotidectomy : a pilot study using ARTISS fibrin sealant. Eur Arch Oto-Rhino-Laryngology . 2019;276(7):2025-2029. doi:10.1007/s00405-019-05449-x

5. Chudek DA, Wilkie MD, Hampton T, Siau R, Panarese A. The effect of fibrin sealant tissue glue in reducing post - operative collections following parotidectomy. Eur Arch Otorhinolaryngol . 2020;277(7):2055-2059. doi:10.1007/s00405-020-05903-1

6. Conboy P BD. Use of Tissue Sealant for Day Surgery Parotidectomy.J Otolaryngol Head Neck Surg . 2008;37(2):208-211. doi:10.2310/7070.2008.0040

7. Mushi E, Kinshuck A, Svecova N, Schache A, Jones TM, Tandon S LJ. The use of Tisseel TM fibrin sealant in selective neck dissection - a retrospective study in a tertiary Head and Neck Surgery centre.Clin Otolaryngol . 2015;40(2):93-97.

8. Bajwa MS, Tudur-Smith C, Shaw RJ SA. Fibrin sealants in soft tissue surgery of the head and neck : A systematic review and meta-analysis of randomised controlled trials. Clin Otolaryngol . 2017;42(6):11411152. doi:10.1111/coa.12837

9. Crossley EJ, Biggs TC, Jog M, Marinakis K, Sipaul F, Brown P ST. Drainless head and neck surgery: A retrospective review of 156 procedures (thyroidectomy, parotidectomy and neck dissections in a tertiary setting): The Southampton experience. Clin Otolaryngol . 2020;Epub ahead. doi:10.1111/coa.13611

10. Al-qahtani K. Initial experience with hemostatic fibrin glue as adjuvant during drainless parotidectomy. Saudi Dent J . 2011;23(2):67-71. doi:10.1016/j.sdentj.2010.11.002

11. Khafif A, Niddal A, Azoulay O, Holostenco V, Masalha M. Parotidectomy via Individualized Mini-Blair Incision. ORL J Otorhinolaryngol Relat Spec . 2020;82(3):121-129. doi:10.1159/000505192

12. Cohen O, Gabay S, Khafif A. Insertion of a Vacuum based Drainer for Post-Parotidectomy Non-resolving Seroma in an Outpatient Setting. Br J Oral Maxillofac Surg . 2020;In Press,. doi:10.1016/j.bjoms.2020.08.047

13. Patel MJ, Garg R RD. Benefits of Fibrin Sealants in Parotidectomy : Is Underflap Suction Drainage Necessary? Laryngoscope . 2006;116(9):1708-1709. doi:10.1097/01.mlg.0000231785.96955.c8

14. Depondt J, Koka VN, Nasser T, Portier F, Guedon C, Barry B GP. Use of fibrin glue in parotidectomy closure. Laryngoscope . 1996;106(6):784-787.

15. Harrison C, Remoundos DD, Harvey KL, et al. Increasing the incidence of drain - free day - case mastectomies with the use of a fibrin tissue sealant ; data from a single surgical center in the United Kingdom.Breast J . 2019;25(6):1090-1096. doi:10.1111/tbj.13419

16. Coniglio AJ, Deal AM HT. Outcomes of drainless outpatient parotidectomy. Head Neck . 2019;41(7):2154-2158. doi:10.1002/hed.25671

17. Ciuman RR, Oels W, Jaussi R DP. Outcome, general, and symptom-specific quality of life after various types of parotid resection. Laryngoscope . 2012;122(6):1254-1261. doi:10.1002/lary.23318

18. Terris DJ, Snyder S, Carneiro-Pla D, Inabnet WB 3rd, Kandil E, Orloff L, Shindo M, Tufano RP, Tuttle RM, Urken M YM. American Thyroid Association Surgical Affairs Committee Writing Task Force. American Thyroid Association statement on outpatient thyroidectomy. Thyroid . 2013;23(10):1193-1202. doi:10.1089/thy.2013.0049

19. Dionigi G, Jun Y, Tufano RP. Transoral endoscopic thyroidectomy via a vestibular approach : why and how ? Endocrine . 2018:275-279. doi:10.1007/s12020-017-1451-x 
20. Choi Y, Lee JH, Kim YH, Lee YS, Chang HS, Park CS RM. Impact of Postthyroidectomy Scar on the Quality of Life of Thyroid Cancer Patients. Ann Dermatol . 2014;26(6):693-699.

21. Rea JL. Partial Parotidectomies : Morbidity and Benign Tumor Recurrence Rates in a Series of 94 Cases. Laryngoscope . 2000;110(6):924-927.

22. Lee SY, Koh YW, Kim BG, Hong HJ, Jeong JH CE. The Extended Indication of Parotidectomy Using the Modified Facelift Incision in Benign Lesions : Retrospective Analysis of a Single Institution. World J Surg 2011 Oct;35(10)2228-37 . 2011;35(10):2228-2237. doi:10.1007/s00268-011-1209-1

23. Findik UY, Topcu SY, Vatansever O. Effects of Drains on Pain , Comfort and Anxiety in Patrents Undergone. Int J Caring Sci . 2013;6(3):412-419.

24. Sethi RK V, Deschler DG. National trends in inpatient parotidectomy : A fourteen-year retrospective analysis. Am J Otolaryngol . 2018;39(5):553-557. doi:10.1016/j.amjoto.2018.06.010

25. Mukdad L, Goel AN, Nasser HB, John MAS. Understanding Nationwide Readmissions After Parotidectomy. Laryngoscope . 2020;130(5):1212-1217. doi:10.1002/lary.28187

Figure Legends:

Figure 1: Example of Fibrin Sealant Use in Excision of deep lobe tumor' Surgical Bed

$1 \mathrm{~A}$ - The surgical bed prior to the deployment of fibrin sealant, the facial nerve is seen in the center of the picture

1B - Application of the FS - filling the entire surgical bed (2 cc Evicel were used)

$1 \mathrm{C}$ - Final result

Figure 2:

Mean hospital length of stay of the fibrin sealant group and the drainage control group

Hosted file

Table 1.pdf available at https://authorea.com/users/407821/articles/518021-drainlessparotidectomy-using-tissue-fibrin-sealant-a-case-control-study

\section{Hosted file}

Table 2.pdf available at https://authorea.com/users/407821/articles/518021-drainlessparotidectomy-using-tissue-fibrin-sealant-a-case-control-study

\section{Hosted file}

Table 3.pdf available at https://authorea.com/users/407821/articles/518021-drainlessparotidectomy-using-tissue-fibrin-sealant-a-case-control-study
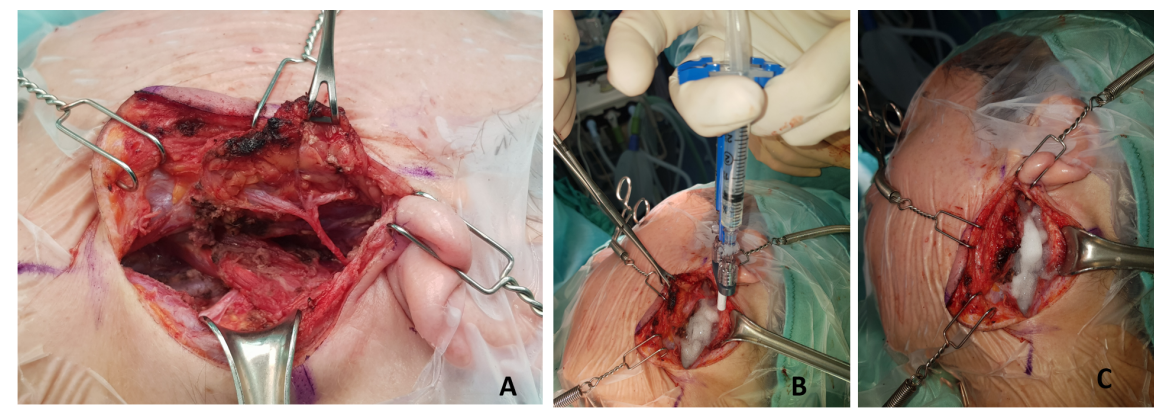


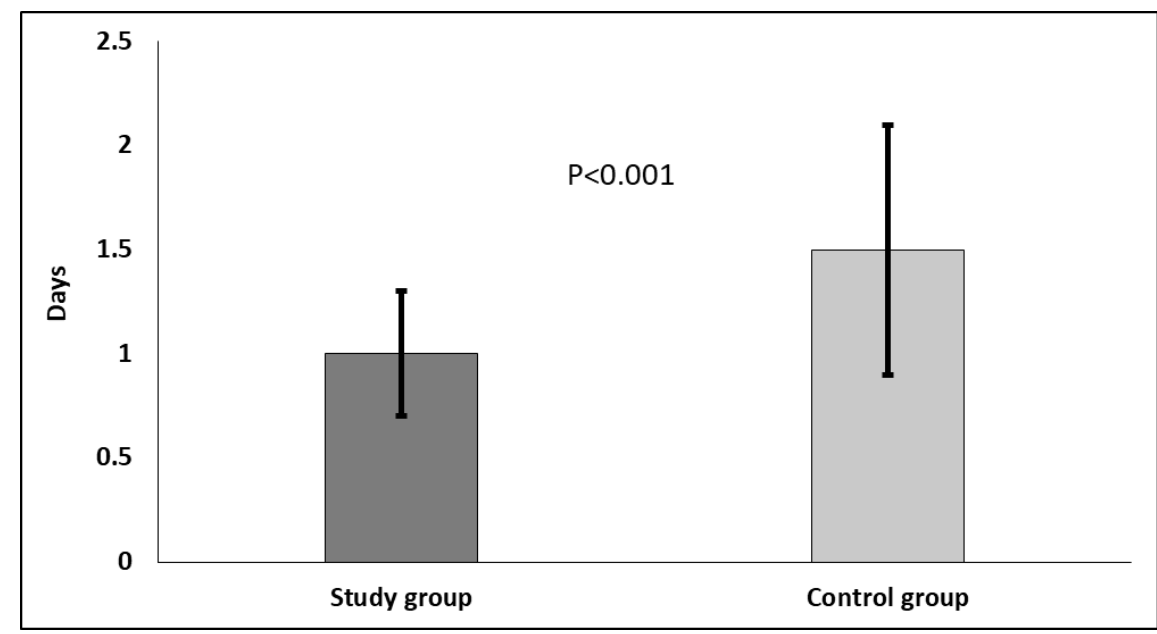

